\title{
Regularization of soft-X-ray imaging in the DIII-D tokamak
}

\author{
A. Wingen, M.W. Shafer, E.A. Unterberg, J.C. Hill, D.L. Hillis \\ Oak Ridge National Laboratory, Oak Ridge, TN 37831, USA
}

\begin{abstract}
An image inversion scheme for the soft X-ray imaging system (SXRIS) diagnostic at the DIII-D tokamak is developed to obtain the local soft X-ray emission at a poloidal cross-section from the spatially line-integrated image taken by the SXRIS camera. The scheme uses the Tikhonov regularization method since the inversion problem is generally ill-posed. The regularization technique uses the generalized singular value decomposition (GSVD) to determine a solution that depends on a free regularization parameter. The latter has to be chosen carefully, and the so called L-curve method to find the optimum regularization parameter is outlined. A representative test image is used to study the properties of the inversion scheme with respect to inversion accuracy, amount/strength of regularization, image noise and image resolution. The optimum inversion parameters are identified, while the Lcurve method successfully computes the optimum regularization parameter. Noise is found to be the most limiting issue, but sufficient regularization is still possible at noise to signal ratios up to 10\%-15\%. Finally, the inversion scheme is applied to measured SXRIS data and the line-integrated SXRIS image is successfully inverted.
\end{abstract}

Keywords:

Tikhonov, regularization, image inversion, tokamak, soft X-ray, DIII-D, GSVD

Email address: wingen@fusion.gat.com (A. Wingen)

Preprint submitted to J. Comput. Phys.

February 10, 2015 


\section{Introduction}

Methods for computed tomography are used in many fields like medicine, nondestructive materials testing, geophysics, atmospheric research and have also been applied to magnetically confined fusion plasmas [1,2]. The common characteristic is that many nonlocal measurements, such as line integrals, are processed mathematically to estimate local physical quantities, such as emissivity or absorption.

Fusion has a long history of Soft x-ray (SXR) detection in the plasma core of tokamaks [3] and stellarators to e.g. detect core MHD modes [4], diagnose core islands [5] or analyze impurity concentrations [6]. A number of tomography methods, which have found applicability to SXR, bolometer, and other tomography diagnostics in magnetically confined fusion plasmas, have been used to various levels of success. Those methods have been limited in spatial resolution and/or cross-detector calibrations [7]. Tangential imaging has historically given better spatial resolution, but interpretation of the images is nontrivial due to the 3-D line-of-sight integration and requires advanced inversion techniques [8, 9], like the Tikhonov regularization [10].

A tokamak confines the plasma inside a torus-shaped vacuum chamber by a strong magnetic field. The magnetic field of a tokamak equilibrium is given by a strong toroidal magnetic field and the poloidal field, generated by the strong toroidal plasma current. The resulting total field is helical in nature; we will exploit this helical property later in the paper. The field lines are organized on surfaces of constant poloidal magnetic flux $\psi$, so called flux surfaces. All field lines on such a flux surface wind themselves helically around the torus and have a constant ratio of toroidal to poloidal rotations, the so called safety factor $q$. Typically $q$ is monotonically increasing from the center towards the separatrix, which is the last closed surface that then determines the plasma boundary and separates the closed field lines inside from the open ones in the so called scrape-off layer. The flux surface configuration is then toroidally axisymmetric. This symmetry allows to project volume quantities, like SXR emission, into a single plane, the tangency plane; we will make use of this later in the paper. Here we focus on the DIII-D tokamak [11].

The soft-X-ray imaging system (SXRIS) [12] at DIII-D is a tangential camera that measures the line-integrated SXR emission in the plasma edge region. The system uses pinhole optics focusing radiation onto a scintillator, which is imaged with visible optics. Is is used to diagnose structural changes due to plasma response [13] to externally applied resonant mag- 
netic perturbations (RMPs). The diagnostic can use different energy filters to discriminate between different spatial regions. Within the steep gradient region of the H-mode pedestal [14], helical displacements of up to $5 \mathrm{~cm}$, which change with the poloidal angle are found, which can be modeled and explained through a synthetic diagnostic [15]. To interpret the measured data, the Tikhonov regularization, found to be applicable to the SXRIS [16], is used to obtain stabilized solutions to the ill-posed problem of inverting line-integrated images.

So far, SXR imaging was used only in the plasma core to detect large scale structures. Here we apply it to the perturbed tokamak plasma edge region for the first time. In this work we determine the requirements for the Tikhonov regularization and develop an algorithm to find high-resolution and high-quality image inversions. The latter are needed to identify and characterize features with potentially low signal to noise and high spatial structure, which are predicted by plasma theory to appear in the perturbed plasma edge.

In the next section we give an overview on the Tikhonov regularization method and construct its solution using the generalized singular value decomposition (GSVD). The method using the GSVD is equivalent to the method developed in Ref. [10] and applied in Refs. [5, 4], but does not require the direct inversion of the side constraint operator. The setup of the SXR image inversion problem is discussed in Sec. 3. The side constraints as well as the optimum regularization parameter is discussed. In Sec. 4 we apply the regularization to an analytic test image and study the inversion quality with respect to noise and resolution. An application to real SXRIS data is demonstrated in Sec. 5. The conclusions summarize the quality of the inversion method and give suggestions on future improvements in the final section.

\section{Mathematical approach to high resolution image inversion}

Mathematically, the 3-D volume of SXR emission is divided into $n=$ $n_{x} n_{y} n_{z}$ emission zones. Each zone is a cube of size $d r^{3}$ inside the volume. On one side of the volume is a pinhole camera, which records the SXR emis-

sion. For simplicity we neglect finite pinhole size effects and assume that the pinhole diameter is infinitesimally small. Note that later on we will reduce the emission volume to 2-D by exploiting a system symmetry. The camera itself is represented by a 2-D "image" which has $m$ number of pixels. 
Each pixel of the image has exactly one line-of-sight through the pinhole and records all emission from the volume along its line-of-sight. Figure 1 shows a schematic sketch of the described setup.

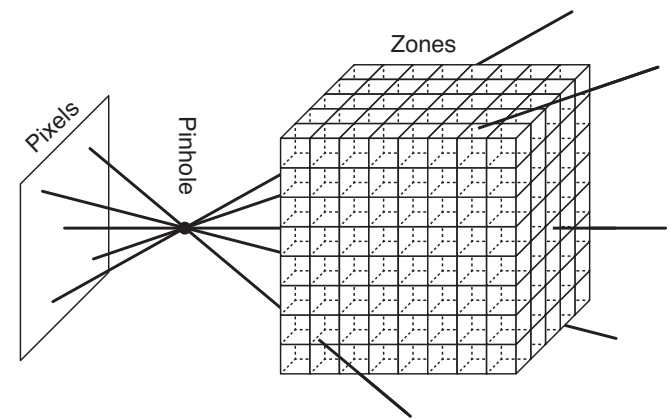

Figure 1: Schematic camera setup for recording line-integrated SXR emission. Several line-of-sights, starting at some camera pixels and going through the pinhole, penetrate the emission volume, which is divided into multiple zones.

The relation between local emission, the emission in each zone of the volume, $\varepsilon \in \mathbb{R}^{n}$ and line integrated emission, the value at each pixel (literally a camera pixel) in the image plane, $\mathbf{s} \in \mathbb{R}^{m}$ is given by the linear equation

$$
\mathbf{L} \cdot \varepsilon=\mathbf{s}
$$

with the geometric transform $m \times n$ matrix $\mathbf{L}$, which will be discussed in more detail in Sec. 3.

\subsection{Tikhonov regularization approach}

In Eq. (1) we use $m>n$ so the system is overdetermined. The solution to this system of equations is given by

$$
\varepsilon_{\mathbf{0}}=\underset{\varepsilon}{\operatorname{argmin}}\|\mathbf{L} \cdot \boldsymbol{\varepsilon}-\mathbf{s}\|,
$$

but the problem is ill-posed.

Regularization is a well known method to find stable solutions to ill-posed problems of such form, which are associated with an ill-conditioned matrix $\mathbf{L}$, i.e. the columns of $\mathbf{L}$ are nearly linear dependent and therefore $\mathbf{L}$ is nearly singular. The solution of an ill-posed problem is in general very sensitive to small perturbations, including numerical rounding errors. A direct inversion of $\mathbf{L}$ or a direct calculation of $\varepsilon$ by standard methods usually result 
in highly inaccurate solutions, which are typically no way near the "real" solution. Replacing the ill-conditioned matrix $\mathbf{L}$ with a well-conditioned matrix, derived from $\mathbf{L}$, does not necessarily lead to a meaningful solution. Regularization provides a stabilization of the solution with respect to small error fluctuations. In the following, we apply the Tikhonov regularization scheme $[17,18,19]$ and instead solve

$$
\boldsymbol{\varepsilon}_{\lambda}=\underset{\varepsilon}{\operatorname{argmin}}\left\|\left(\begin{array}{c}
\mathbf{L} \\
\lambda \mathbf{C}
\end{array}\right) \varepsilon-\left(\begin{array}{c}
\mathbf{s} \\
\lambda \mathbf{C} \cdot \varepsilon^{*}
\end{array}\right)\right\|,
$$

where $\|\mathbf{x}\|=\left[\sum x_{i}^{2}\right]^{1 / 2}$ is the Euclidean norm and $\lambda$ is the free regularization parameter which controls the side constraint $\min \left\|\lambda \mathbf{C} \cdot\left(\varepsilon-\varepsilon^{*}\right)\right\|$. $\varepsilon^{*}$ is an a priori assumption about the solution itself, which can either be zero, i.e. no assumption is made (used throughout the paper), or some assumed local emission distribution within the zones, which is then forced onto the solution.

\subsubsection{Side constraint}

$\mathrm{C} \in \mathbb{R}^{n \times n}$ is an operator which represents the side constraint. It is a necessary contribution to the actual ill-posed minimization problem to find a meaningful solution. Numerically, a minimizer $\varepsilon_{0}$ of Eq. (2), using $\lambda=0$, can be found, but for ill-posed problems it is typically highly oscillatory (see e.g. Fig. 6(a)) or far away from the "real" solution. The side constraint can keep the obtained minimum solution within specified bounds, can force a continuous solution or can ensure other problem-specific properties. If there is no other obvious side constraint to the considered problem, $\mathbf{C}$ is often used as either the identity matrix $\mathbf{I}_{n}$ or a derivative operator like the Laplace operator. In case of $\mathbf{C}=\mathbf{I}_{n}$ the side constraint forces the norm of the solution to be small and therefore ensures a bounded solution. A derivative operator on the other hand limits gradients within the solution and ensures a certain level of smoothness.

\subsubsection{Choice of regularization parameter}

The parameter $\lambda$ determines the "strength" of the regularization, i.e. the weight of the side constraint, which is given by $\mathbf{C}$, relative to the solution constraint, Eq. (2). For $\lambda=0$ the solution $\varepsilon_{\lambda}$ is unregularized and therefore, as it is typical for ill-posed problems, undamped with high frequency components, although the residual $\left\|\mathbf{L} \cdot \varepsilon_{\lambda}-\mathbf{s}\right\|$ is very small. Very large values of $\lambda$ on the other hand lead to $\varepsilon_{\lambda}$ which are no longer meaningful solutions to the 
actual problem since the residual becomes arbitrarily large. It is imperative to find the optimum regularization parameter, which is a non-trivial task. Several methods are known to literature to estimate the optimum regularization parameter [20]; here we apply the L-curve criterion [21], as further discussed in Sec. 3.2.

\subsubsection{Generalized singular value decomposition}

To solve Eq. (3), we use the generalized singular value decomposition (GSVD)

$$
\begin{array}{r}
\mathbf{U}^{\mathbf{T}} \cdot \mathbf{L} \cdot \mathbf{Z}=\operatorname{diag}(\boldsymbol{\sigma}) \\
\mathbf{V}^{\mathbf{T}} \cdot \mathbf{C} \cdot \mathbf{Z}=\operatorname{diag}(\boldsymbol{\mu})
\end{array}
$$

with the orthonormal matrices $\mathbf{U} \in \mathbb{R}^{m \times n}$ and $\mathbf{V} \in \mathbb{R}^{n \times n}$, the non-singular matrix $\mathbf{Z} \in \mathbb{R}^{n \times n}$ and the diagonal matrices $\operatorname{diag}(\boldsymbol{\sigma})$ and $\operatorname{diag}(\boldsymbol{\mu})$, both $\in$ $\mathbb{R}^{n \times n}$ with

$$
\begin{aligned}
& 0 \leq \sigma_{1} \leq \ldots \leq \sigma_{n} \leq 1 \\
& 1 \geq \mu_{1} \geq \ldots \geq \mu_{n} \geq 0
\end{aligned}
$$

and

$$
\boldsymbol{\sigma}^{T} \cdot \boldsymbol{\sigma}+\boldsymbol{\mu}^{T} \cdot \boldsymbol{\mu}=\mathbf{I}_{n}
$$

Then the ratios

$$
\gamma_{i}=\frac{\sigma_{i}}{\mu_{i}}, i=1 \ldots n
$$

are the generalized singular values of the matrix pair $(\mathbf{L}, \mathbf{C})$. Note that for $\mathbf{C}=\mathbf{I}_{n}$ the GSVD becomes the standard SVD.

In Ref. [10] the regularized solution is constructed by using the standard SVD of the Matrix $\mathbf{L C}^{-1}$. By using the definitions in Eq. (4) we find

$$
\mathbf{L} \cdot \mathbf{C}^{-\mathbf{1}}=\mathbf{U} \cdot \operatorname{diag}(\boldsymbol{\gamma}) \cdot \mathbf{V}^{\mathbf{T}}
$$

which is identical to the definition in the reference. Therefore, the regularized solution constructed here is equivalent to the one using the SVD of $\mathbf{L C}^{-\mathbf{1}}$. The difference is that the GSVD method does not require the direct inversion of the operator $\mathbf{C}$.

Using the GSVD we define

$$
\boldsymbol{\beta}=\mathbf{U}^{T} \cdot \mathbf{s}, \quad \boldsymbol{\omega}=\mathbf{Z}^{-1} \cdot \varepsilon^{*}
$$


and find for the regularized solution

$$
\varepsilon_{\lambda, i}=\sum_{j=1}^{n} Z_{i j} f_{j}\left[\frac{\beta_{j}}{\sigma_{j}}+\frac{\lambda^{2}}{\gamma_{j}^{2}} \omega_{j}\right]
$$

with the filter factors

$$
f_{j}=\frac{\sigma_{j}^{2}}{\sigma_{j}^{2}+\lambda^{2} \mu_{j}^{2}}=\frac{\gamma_{j}^{2}}{\gamma_{j}^{2}+\lambda^{2}} .
$$

\section{Setup of image inversion in tokamaks}

We aim to determine the local SXR emission inside a tokamak plasma from SXR camera measurements as sketched by Fig. 1. The actual setup is shown in Fig. 2(a), which is a top down view of the tokamak cross-section. The pinhole is located at the outer wall, while the camera views tangentially into the torus; the view cone is given by the grey area. To achieve meaningful statistics of local emission we need a sufficiently high number of different lineof-sights to penetrate the emission volume. This number of pixels should be larger than the number of zones, i.e. $m>n$. Note that the Tikhonov regularization can treat underdetermined cases with $m<n$ as well, but we do not consider such cases here. Since the emission volume is $3-\mathrm{D}$ while the pixel plane is 2-D, maintaining $m>n$ and simultaneously achieving a reasonable resolution of local emission can easily become computationally challenging. In a tokamak the helical symmetry of the equilibrium field line configuration can be exploited.

Figure 2(b) shows a poloidal cross-section in DIII-D; it is the tangency plane of the camera's optical axis marked by the green cross. The separatrix and multiple flux surfaces are given, indexed by the poloidal flux which is normalized to 1 at the separatrix and 0 at the magnetic axis in the plasma center. The flux surface configuration as shown in Fig. 2(b) is toroidally axisymmetric but the field lines are helical. The view cone of the camera covers the grey highlighted area.

To make use of the helical field line symmetry, we imply the fundamental assumption that SXR emission is constant along magnetic field lines for the length of the trace in Fig. 2(c), which is between $0 \mathrm{~m}$ and $1.2 \mathrm{~m}$ along a field line. This assumption is reasonable in the SXR emission regime, because thermal electrons follow field lines quite closely [22] and parallel transport 

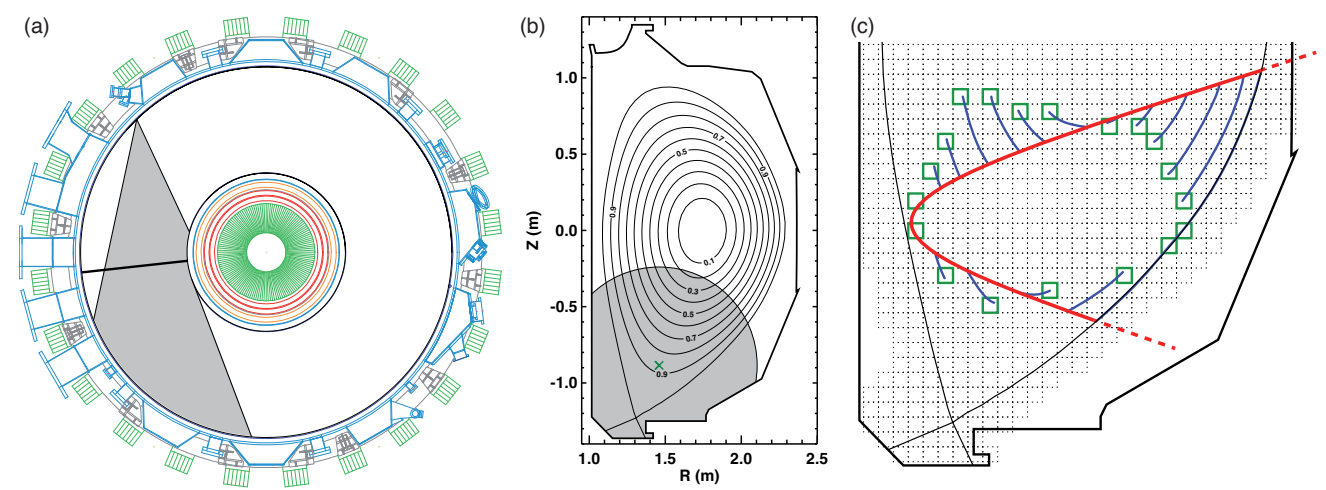

Figure 2: (color online) (a) Schematic top down view of the tokamak. The grey area shows the view cone of the pinhole camera. The line across it represents the tangency plane of the optical axis.

(b) The poloidal cross-section of the tangency plane in DIII-D. The grey circle represents the area seen by the camera. The green cross is the center, where the optical axis intersects. The separatrix and multiple plasma equilibrium flux surfaces are shown.

(c) A magnification of the area viewed by the camera, divided into emission zones. The red line is the toroidal projection of a line-of-sight into this poloidal cross-section. Various points along the line-of-sight are traced along the magnetic field lines (blue traces), based on their helicity, to the respective emission zones (green boxes) in the tangency plane, which are then the source of emission along this line-of-sight.

is much larger than perpendicular, while electron density and electron temperature are assumed constant on flux surfaces in standard axisymmetric equilibrium reconstructions. Note, that we do not need to assume constant SXR emission on flux surfaces here. So, as shown in Fig 2(c), we step along a line-of-sight (the red line is the toroidal projection of it into the tangency plane) and trace each point along a magnetic field line towards the tangency plane. Such traces are shown by the blue lines in the figure. The intersections of the various traces with the tangency plane then give the respective emission zones, which are represented by the green boxes within the black dashed zone grid. By this procedure we can reduce the emission volume by one dimension to the 2-D tangency plane, which is divided into a 2-D grid of $n=n_{R} n_{Z}$ square emission zones with resolution $d R=d Z=h=$ const. The zone grid is thereby limited by the vessel wall at the bottom, by the $\psi=1.1$ scrape-off-layer surface at the left and right and by the uppermost line-of-sight at the top.

The geometric transform, or $L$-Matrix, is then built up row by row, start- 
ing out as a row of zeros. Each row represents one line-of-sight, i.e. one pixel, while each column represents one emission zone. A line-of-sight is discretized with a step size $d s$ which should be smaller than the zone resolution $d R$ to get reasonable statistics. For each point along the line-of-sight $i$ the respective emission zone $j$ is located by following an equilibrium field line. Then the value of the emission zone $i, j$ is increased by one, i.e. $L_{i, j}=L_{i, j}+1$. Note that the resulting matrix is sparse in nature.

\subsection{Choice of side constraint}

In the case of SXR emission in DIII-D discharges, we expect to see high emission from within the plasma, low or even no emission outside and possibly a steep gradient at the plasma edge. So we neither want to limit the solution itself nor its gradients, i.e. the first-order spatial derivative. On the other hand, we want to suppress high frequency fluctuations, like a noisy fragmentation, in the solution. So, we use the Laplacian operator, the second-order spatial derivative, to ensure that the solution is sufficiently smooth, while $\lambda$ controls the amount of smoothness. Let $\varepsilon_{i, j}=\varepsilon\left(R_{i}, Z_{j}\right), i=0, \ldots, n_{R}-1$ and $j=0, \ldots, n_{Z}-1$, be an emission zone of the grid in Fig. 2(c) with the zone size $h$ in both dimensions, then we approximate the Laplacian operator by the second order finite difference scheme

$$
\left(\partial_{R}^{2}+\partial_{Z}^{2}\right) \varepsilon_{i, j}=\left(\varepsilon_{i+1, j}+\varepsilon_{i-1, j}-4 \varepsilon_{i, j}+\varepsilon_{i, j+1}+\varepsilon_{i, j-1}\right) / h^{2}
$$

and with $k=j * n_{R}+i=0, \ldots, n-1$, we define the $n \times n$ Matrix

$$
C_{k, k}=-4 \quad C_{k, k \pm 1}=1 \quad C_{k, k \pm n_{R}}=1
$$

and zero elsewhere. Inside the grid, the elements of $\mathbf{C}$ are well defined, but not at the boundary, because elements like $C_{0,-1}$ obviously do not exist. So, we have to define additional boundary conditions for the emission $\varepsilon_{i, j}$. There are two common choices for non-periodic cases:

1. Dirichlet boundary conditions, which set $\varepsilon_{i, j}=0$ if $\left(R_{i}, Z_{j}\right)$ is outside the grid. Then $\mathbf{C}$ is given by Eq. (13) with both indices between 0 and $n-1$.

2. Von Neumann boundary conditions, which set the first-order derivative of $\varepsilon$ to zero at the boundary. Using the symmetric finite difference for the first-order derivative, we find that $\varepsilon_{-1, j}=\varepsilon_{1, j}$ and $\varepsilon_{i,-1}=\varepsilon_{i, 1}$. In those cases, the respective elements of $\mathbf{C}$ are set to 2 instead of 1 . 
In the following we use Dirichlet boundary condition, which are obviously appropriate and well satisfied at the left, right and lower boundary. At the upper boundary von Neumann boundary conditions would be the better choice, but, as we will discuss later in the paper, the upper boundary suffers from very low precision and is disregarded anyway. Using the von Neumann boundary condition at the upper boundary was checked numerically and gives the same (poor) result as the Dirichlet condition.

\subsection{L-curve}

For a given regularization parameter $\lambda$ and its regularized solution $\varepsilon_{\lambda}$, as obtained by the Tikhonov method, we define the residual norm

$$
\rho^{2}=\left\|\mathbf{L} \cdot \boldsymbol{\varepsilon}_{\lambda}-\mathbf{s}\right\|^{2}=\lambda^{4} \sum_{i=1}^{n}\left(\frac{\beta_{i}-\sigma_{i} \omega_{i}}{\gamma_{i}^{2}+\lambda^{2}}\right)^{2}
$$

and the semi-norm

$$
\eta^{2}=\left\|\mathbf{C} \cdot\left(\varepsilon_{\lambda}-\varepsilon^{*}\right)\right\|^{2}=\sum_{i=1}^{n}\left(\gamma_{i} \frac{\beta_{i}-\sigma_{i} \omega_{i}}{\gamma_{i}^{2}+\lambda^{2}}\right)^{2} .
$$

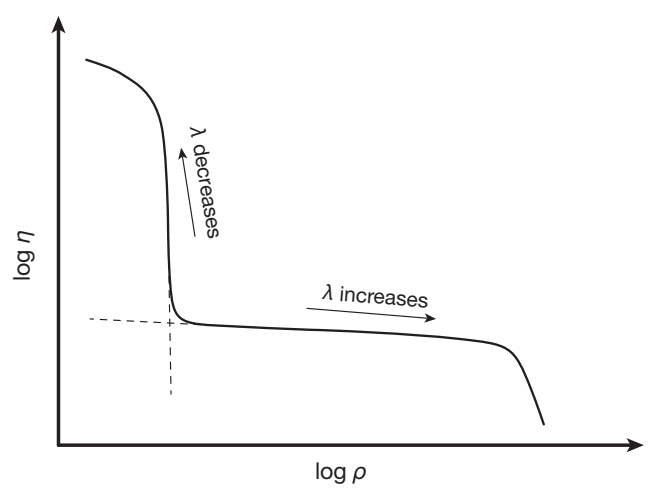

Figure 3: Schematic of a typical L-curve

One method to determine the optimum regularization parameter $\lambda$ is to plot the semi-norm versus the residual norm for various values of $\lambda$ on a double log scale, as shown by the schematic in Fig 3. The resulting curve has the typical form of an "L", hence the name L-curve. The optimum point of operation is at the corner of the L-curve, which is the best trade off between 
solving the problem and satisfying the side constraint. Increasing $\lambda$ also increases the level of regularization, i.e. oversmooths the solution in case of a Laplacian operator; the residual norm strongly increases. Decreasing $\lambda$ on the other hand reduces the regularization, i.e. the solution becomes more noisy and the semi-norm rises.

\subsubsection{Optimum regularization parameter}

Here we use the L-curve to determine the optimum regularization parameter by localizing the L-curve corner. Besides assessing the L-curve visually, there are two typical ways of localizing the L-curve corner numerically. First, the corner of the L-curve represents a local maximum in the the L-curve's curvature, as given by

$$
\kappa=\frac{\eta^{\prime \prime}(\rho)}{\left(1+\eta^{\prime}(\rho)^{2}\right)^{3 / 2}},
$$

and second, at the corner the distance of the L-curve from the origin $\sqrt{\rho^{2}+\eta^{2}}$ is locally minimal. Both points should coincide, but in a practical application this is not necessarily the case because $\eta$ contains a hidden free scaling parameter $\alpha \neq 0$. Considering two regularizations as given by Eq. (3), indexed '1' and '2' respectively, with

$$
\begin{gathered}
\mathbf{L}_{1}=\mathbf{L}_{2}, \quad \mathbf{s}_{1}=\mathbf{s}_{2}, \quad \varepsilon_{1}^{*}=\varepsilon_{2}^{*}, \\
\mathbf{C}_{1}=\alpha \mathbf{C}_{2}, \quad \lambda_{1}=\frac{1}{\alpha} \lambda_{2},
\end{gathered}
$$

then obviously leads to

$$
\varepsilon_{\lambda, 1}=\varepsilon_{\lambda, 2}
$$

From Eqs. (14) and (15) we find

$$
\rho_{1}=\rho_{2}, \quad \eta_{1}=\alpha \eta_{2} .
$$

Therefore we use both criteria to determine $\alpha$, so that the point of maximum curvature coincides with the point of minimal distance. The corresponding $\rho$ then gives the optimum regularization parameter. Empirically we find that $\alpha$ scales $\mathbf{C}$ in such a way that $O(\alpha\|\mathbf{C}\|) \approx O(\|\mathbf{L}\|)$, i.e. the Frobenius norm of both matrices are in the same order of magnitude. 


\section{Image inversion quantification}

To test and quantify the implemented regularization scheme, we first apply it to an analytic test image. The structures in the test image reflect features one could expect to see in a real discharge application. The test image inversion consists of several steps:

1. Generate analytically a 2-D image of local emission at the tangency plane, as described in the next section. This 2-D image is numerically represented by the vector $\varepsilon_{0}$.

2. Construct the geometric transform $\mathbf{L}$ and obtain the line integrated test SXR emission $\mathbf{s}=\mathbf{L} \cdot \boldsymbol{\varepsilon}_{0}$.

3. Apply the Tikhonov regularization to reconstruct the local emission at the tangency plane $\varepsilon_{\lambda}$ and determine the optimum regularization parameter $\lambda_{\text {opt }}$ through the L-curve.

4. Compare the regularized solution $\varepsilon_{\lambda_{\text {opt }}}$ with the analytic test image $\varepsilon_{0}$ to quantify the quality of the inversion.

As a measure, a figure of merit or FOM is defined inside the zone grid

$$
e_{F O M}=\frac{\left\|\varepsilon_{\lambda_{o p t}}-\varepsilon_{0}\right\|}{\left\|\varepsilon_{0}\right\|},
$$

which is the relative error, given in $\%$, of the regularized solution with respect to the known, exact solution. The four steps are illustrated in more detail below.

\subsection{Test image development and characterization}

To create a representative test image, we use a typical magnetic field configuration in DIII-D, consisting of the axisymmetric equilibrium field plus a non-axisymmetric small perturbation field, and assign SXR emission to the field line penetration depth structure via an empirical emissivity profile $\varepsilon(\psi)$. Then we smooth the resulting 2-D emission by a simple moving average filter. The procedure of creating a test image is similar to the SXR modeling efforts described in Ref. [15].

The results of the inversion steps 1-3 can be seen in Fig. 4. Figure 4(a) shows the created test image $\varepsilon_{0}$. Note that the image is limited to the emission zones covered by the geometric transform. The test emission shows multiple different features, which we also would like to resolve in a real discharge application. The most obvious feature is the plasma bulk, given by 


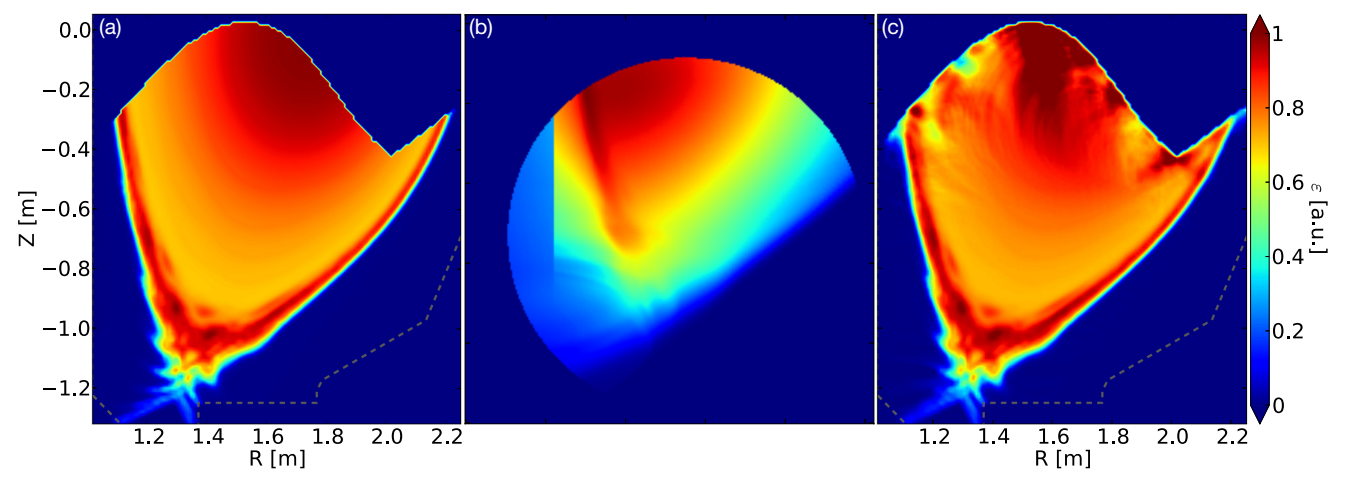

Figure 4: (color online) (a) Test image of local emission

(b) Line integrated emission image as seen by SXR camera. Created from (a).

(c) Local emission obtained through image inversion of (b) by Tikhonov regularization with $\lambda=1.9$

any emission greater than zero. The plasma bulk is characterized by a coarse structure; the emission peaks along the edge, decreases further inwards and then increases again in the core. On top of the coarse structure, several different fine-structure features appear due to the perturbation. One feature is the boundary shape, showing several so called lobes, that reach out towards the vessel wall at the lower corner of the separatrix. Inside the plasma edge small regions of increased emission can be found. Those features are created by islands in the magnetic structure.

The line integrated test emission as obtained from the test image is shown in Fig. 4(b). Many of the test image's features are obscured due to the line integration. Note that the line integrated image is limited by the circular detection plane, while the emission inside is limited by the vessel wall. The centerpost, which is the vertical wall segment on the left side in Fig. 2(b), is clearly visible by the vertical cut-off in the line integrated emission. For the remainder of Sec. 4, Fig. 4(b) is the starting image $\mathbf{s}$ for the Tikhonov regularization.

The optimum regularized solution $\varepsilon_{\lambda_{\text {opt }}}$ can be seen in Fig. 4(c). All features of the original test image, which is the exact solution to the regularized problem, are well reconstructed in $\varepsilon_{\lambda_{\text {opt }}}$, not only the plasma bulk, but also the lobes and the islands. Only at the very top, in the core region, there are differences between $\varepsilon_{\lambda_{\text {opt }}}$ and $\boldsymbol{\varepsilon}_{0}$. These deviations are artifacts of the regularization, which are systematic and therefore unavoidable without additional a-priori information. The upper boundary of the emission zone grid 
is defined by the limit of line-of-sight coverage. This implies that, compared to the lower part of the zone grid, only very few line-of-sights intersect with emission zones at the upper boundary and therefore the uncertainty becomes large in this region; hence artifacts appear.

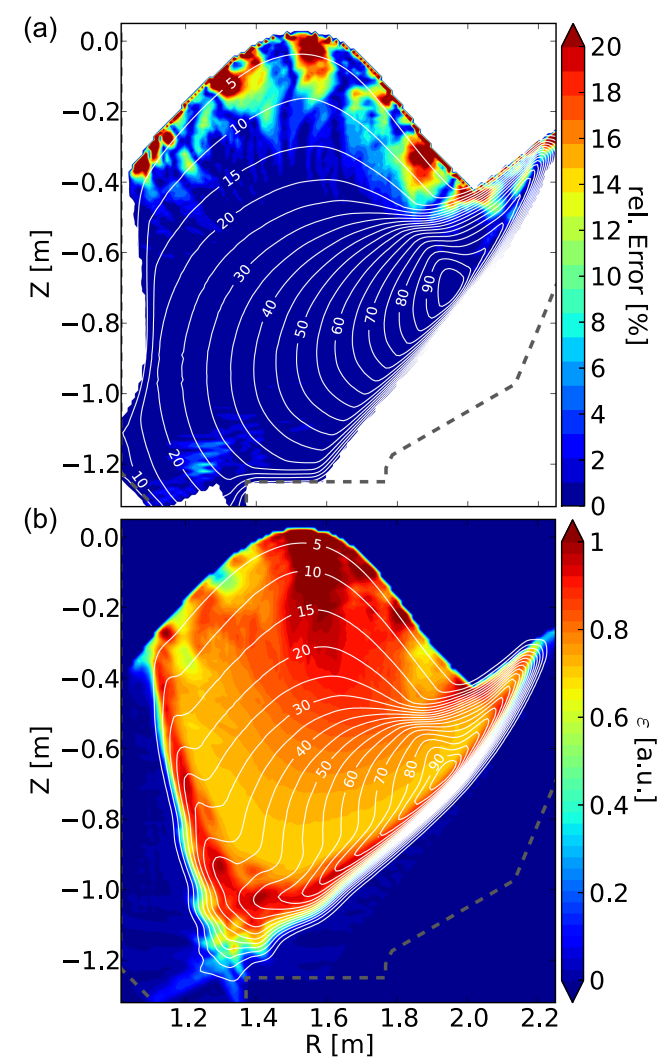

Figure 5: (color online) (a) Relative error in percent between Fig. 4(a) and (c). The white lines give the level of precision. The white area is outside the emission zone grid.

(b) Same as Fig. 4(c), but the white lines give the level of certainty in percent of the inverted image.

Figure 5(a) shows the relative difference between the test image and the optimum regularized solution in percent inside the emission zone grid. The number of sight-line intersections each with emission zone is a measure of the so called precision

$$
P_{j}=\sum_{i=1}^{m} L_{i, j}, \quad j=1, \ldots, n
$$


one can expect from sight-line coverage. The white lines are the precision contours, given as percentage of its maximum, which clearly reflect the original view cone of the pinhole camera, projected along the helical symmetry into the poloidal cross-section. The artifacts start to appear outside the $15 \%$ level and become prominent outside the $10 \%$ level. The only exception appears to be the upper right extension of the zone grid, which we will neglect in the following, since it does not cover any significant area of the plasma anyway. So we conclude that precision levels of at least $15 \%$ are sufficient for a reliable image inversion.

Figure 5(b) is the same as Fig. 4(c), but with the contours of the so called

certainty $A_{j}=\varepsilon_{\lambda_{\text {opt }}, j} P_{j}$ overlayed as white lines. The latter is normalized to its maximum value and therefore given in percent. The certainty becomes large in areas that have a sufficient precision and a signal that is relevant compared to a possible noise floor. By comparing to (a) we see that a certainty level of about $15 \%$ or above eliminates the artifacts and covers the entire internal fine structure while leaving out the scrape-off layer as well as the smallest lobe structures due to their very low emission.

In practice we are primarily interested in SXR emission from the perturbed structures in the plasma edge, so we limit the evaluation of the FOM to the region below $Z=-0.6$. Except for a small region in the lower left corner of Fig. 5(a), where the relative error rises locally up to about $8 \%$ due to some deviations in the fine structure of the lobes, the relative error is negligible throughout the considered region. We find $e_{F O M}=2.5 \%$ for the regularized image. This confirms the good reconstruction of the test image.

\subsection{Parameter dependencies of regularization method}

The inversion scheme and therefore the quality and success of the regularized image depends on multiple parameters. The most obvious one is of course the regularization parameter $\lambda$ itself. But also other parameters are involved, which potentially can influence the regularization quality significantly. In this section we will discuss the various parameters and their impact on the inversion quality.

\subsubsection{Regularization parameter $\lambda$}

Since we obtained the line integrated test emission $\mathbf{s}$ in the last section (Fig. 4(b)) directly through the geometric transform $\mathbf{L}$, the test image $\varepsilon_{0}$ is the exact analytic solution to Eq. (1). To study the effectiveness of the inversion scheme in a closer approximation to a practical application, we 
convert the test problem setup to an actual minimization problem as Eq. (2) by adding a small level of random white noise to $\mathbf{s}$ as a perturbation; here we use $2 \%$ of the maximum signal.
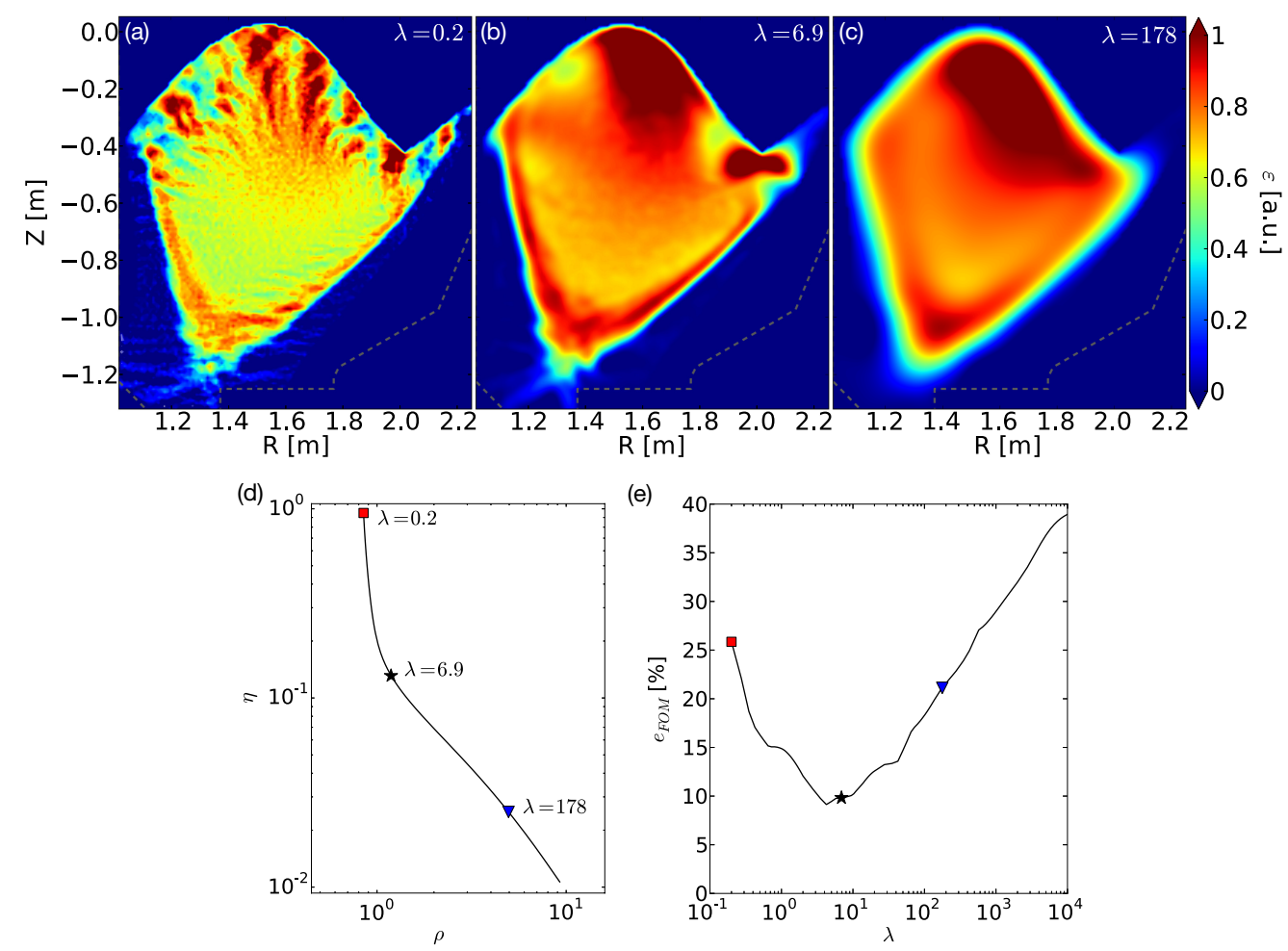

Figure 6: (color online) Tikhonov regularization of Fig. 4(b) with $2 \%$ noise added.

(a) Regularized image for $\lambda=0.2$.

(b) Regularized image for the optimum regularization parameter $\lambda=6.9$.

(c) Regularized image for $\lambda=178$.

(d) The L-curve. The three markers show the position of solutions (a)-(c) on the L-curve.

(e) Figure of Merit evolution for the full range of regularization parameters used in (d). Again the markers give the FOMs for the solutions (a)-(c).

Figure 6 shows three different regularized solutions for three different regularization amounts $\lambda$. First, a strongly under-regularized solution for $\lambda=0.2$ in Fig 6(a), which is characterized by high frequency oscillations and fragmentation. Second, the optimum solution according to the L-curve criterion for $\lambda=6.9$ in Fig 6(b), which shows good agreement with the test image, and third, a strongly over-regularized solution for $\lambda=178$ in Fig 6(c), which is over-smoothed and smears out any structure besides the 
overall plasma bulk itself. The corresponding L-curve is shown in Fig 6(d), which is not as strongly shaped as suggested by the theoretical curve in Fig 3. Nevertheless, the algorithm described in Sec. 3.2.1 successfully located the L-curve corner. This is verified by the FOM, Eq. (21), as shown in Fig 6(e). The L-curve corner, given by the black star marker is also located very closely to the minimum of FOM, which means that the corresponding solution, Fig 6(b), gives the best possible fit with the test image at $e_{F O M}=$ $9.8 \%$. This value is already larger than the FOM of the case without noise, but still much smaller than the FOMs of Fig 6(a)\&(c), which are at $25.8 \%$ and $21.1 \%$ respectively.

\subsubsection{Effect of noise and resolution on inversion quality}

In the last section, we added a small amount of white noise to the line integrated test emission and found that noise has a significant influence on the inversion quality. Potentially, noise can prevent the resolution of the fine structure or even disable the inversion completely. To study the impact of noise on the inversion, we add different levels of random white noise, given in $\%$ of the maximum signal, to the line integrated SXR emission in Fig. 4(b). The white noise is given by evenly distributed random values between 0 and 1 , multiplied by the noise/signal ratio. Here we use 1\%,2\%,5\%,10\% and $20 \%$. Higher levels of noise are less relevant here, because at $20 \%$ noise the fine structure can no longer be recovered; only the plasma bulk remains. Note that after the addition of noise any negative signal is set to zero (e.g. in the scrape-off layer), since negative emission is non-physical.

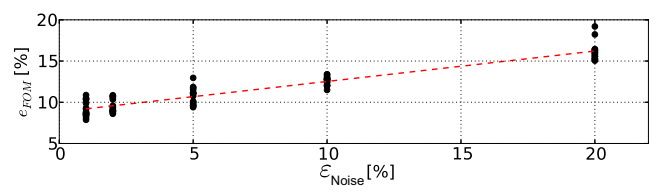

Figure 7: (color online) Figure of merit (FOM) in dependence of noise level for the optimal $\lambda$ respectively. The red line is a linear regression through the data points.

Figure 7 shows the dependency of the FOM on the applied noise level. It shows that the FOM increases quite linearly with the noise level over the considered range. Note that there are multiple data points at each noise level. Those are for different pixel resolutions and step sizes along the sight line, while the zone resolution is kept fixed at $100 \mathrm{~m}^{-1}$. The reason for this becomes clear in the next section. 
The main conclusion which can be drawn from this analysis is that noise should be reduced as much as possible before the image inversion. But since some noise level is unavoidable, we can see that a reasonable reconstruction is still possible up to a noise/signal level of 10\%-15\%. Noise reduction through various filter techniques is an important part of image processing, and future work will test the effects such conditioning methods have on the resulting inverted image.

Besides random noise, systematic errors can also limit the inversion quality by loss of information or the appearance of artifacts. Such systematic errors occur due to uncertainties in the positioning of the pixels, the distance of the pixels to the pinhole, the angle of the camera and other camera geometries. Those effects are not included in our test image analysis. But, for an actual diagnostic, like the SXRIS, it is extremely important, and non-trivial to minimize the systematic errors by precise positioning and calibration.

Further parameters are the resolution of the line integrated emission as given by the number of pixels; the spatial resolution of the local emission in the tangency plane as given by the number of zones per meter; and the number of steps along each line-of-sight at which we map the line-of-sight along the helical symmetry to the tangency plane, in the following just referred to as steps (see the blue lines in Fig. 2(c)) per meter.

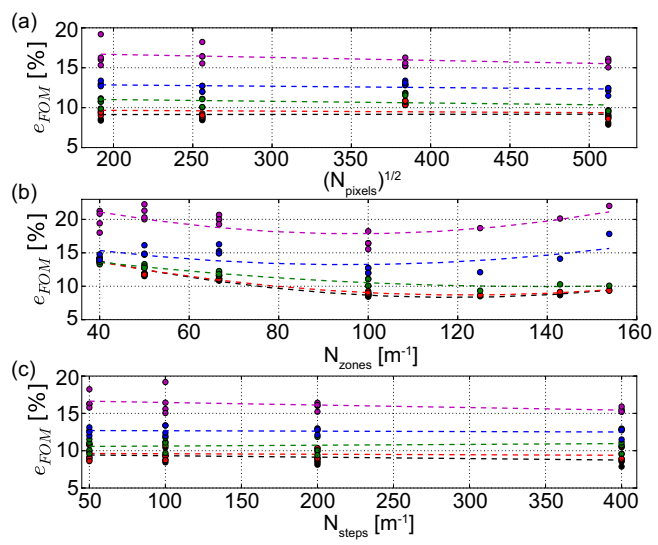

Figure 8: (color online) (a) Figure of merit (FOM) in dependence of pixel resolution. The various colors indicate different noise levels: black $=1 \%$, red $=2 \%$, green $=5 \%$, blue $=$ $10 \%$ and purple $=20 \%$.

(b) same as (a) but for zone resolution.

(c) same as (a) but for step resolution.

In Fig. 8 we show the dependencies of FOM on the various resolutions at 
different noise levels. First, we find that neither the pixel resolution nor the step resolution have much impact on the FOM within the considered range. Keep in mind that we already set the limitations that the number of pixels must be larger than the total number of zones and that the number of steps must be larger than the number of zones. These limits are sufficient, so that at low to medium noise levels and at a fixed number of zones the number of pixels and steps can be minimized for computational efficiency. At high noise levels though we see a weak reduction of FOM with pixel and step number.

The zone resolution on the other hand shows a large impact on the FOM, which becomes minimal roughly at $100-120 \mathrm{~m}^{-1}$ for all noise levels. To help find the optimum zone resolution, we calculated a 2-D contour map of FOM versus number of zones and noise, shown in Fig. 9. As we can see, the FOM has a minimum valley along the noise axis, as indicated by the white line. The white line gives the optimum zone resolution for each noise level. There is some variation of the zone number along the white line, but essentially a value between $100 \mathrm{~m}^{-1}$ and $120 \mathrm{~m}^{-1}$ is preferred.

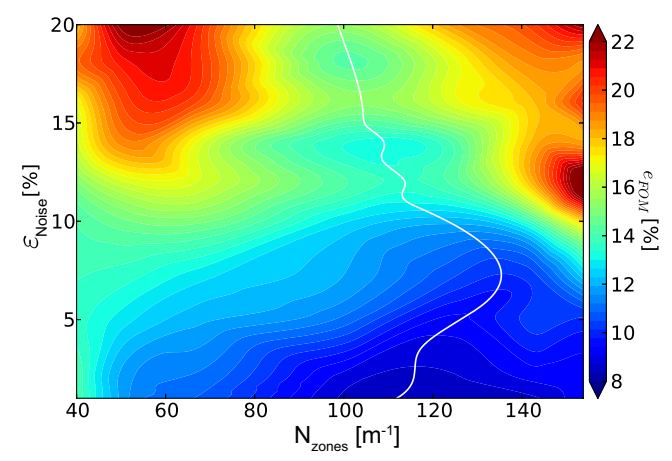

Figure 9: (color online) Figure of merit (FOM) in dependence of noise level and zone resolution. The white line is the FOM minimum with respect to zone resolution at each noise level.

Based on this analysis and available computational resources we choose the following set of parameters for the application to the DIII-D SXR imaging system (SXRIS), discussed in Sec.5. We set our zone resolution to $100 \mathrm{~m}^{-1}$, since we are yet uncertain about the actual noise level in the SXRIS images. The chosen value is optimal for higher noise levels, but still sufficient for lower levels. Based on the system dimensions and the percentages of relevant pixels and zones within the grids, we can derive the empirical formula

$$
0.52 N_{\text {pixels }}>0.44 s_{R} s_{Z} N_{\text {Zones }}^{2},
$$


with $s_{R}=1.36 \mathrm{~m}$ and $s_{Z}=1.75 \mathrm{~m}$ the size of the zone grid, to find the least number of pixels required to satisfy $m>n$ in the L-Matrix. Note that zones or pixels with no signal do not contribute to the L-Matrix. Note also that, through downsampling, the number of pixels can be chosen freely up to the limit of the camera resolution. Since there is a weak dependence on the number of pixels at higher noise level, we use $N_{\text {pixels }}=256 \times 256$ and have $m=34078$ and $n=10472$. The minimum number of steps required is $100 \mathrm{~m}^{-1}$. Since the number of steps has the least impact on computational resources, we choose $N_{\text {steps }}=200 \mathrm{~m}^{-1}$ here.

\section{Application to a measured SXR image}

In the following section, we apply the Tikhonov regularization to the SXRIS diagnostic at DIII-D to spatially invert the line-of-sight integrated SXR images, taken by the diagnostic, to the tangency plane. The diagnostic setup is shown in Fig. 2. For further technical details about the SXRIS diagnostic, see Ref. [12]. In this work, we use a typical H-mode discharge with RMPs. A Beryllium high-pass filter with an energy cut-off at about $600 \mathrm{eV}$ is used in the SXRIS which removes low energy ultra-soft X-ray emissions from outside the plasma and from wall interactions. The measured emission generally comes from the steep gradient region of the plasma edge, which means, we expect the inverted SXR image to show similarities to the test image we used in the previous section.

The measured SXR emission data from the SXRIS camera is shown in Fig. 10(a). Note that the data is not only spatially integrated along the view cone, but also temporally over an integration time of $75 \mathrm{~ms}$, while our algorithm inverts the spatial line-of-sight integration. So, the regularized image is still temporally integrated. We apply the image inversion scheme to Fig. 10(a), given as

1. Calculate the geometric transform $\mathbf{L}$, as discussed in Sec. 3;

2. Decompose L into the GSVD (Eq. (4)), using the Laplacian operator C (Eq. (13)) with Dirichlet boundary conditions;

3. Evaluate the Tikhonov regularization (Eq. (10)) for 120 different $\lambda$ values on a log scale within the range $0.2 \leq \lambda \leq 2 \times 10^{4}$ and determine the optimum $\lambda$ through the L-curve method, as discussed in Sec. 3.2.1;

4. Evaluate the Tikhonov regularization for the optimum $\lambda$ and obtain the regularized solution $\varepsilon_{\lambda_{\text {opt }}}$, shown in Fig. 11. 
Note that the computationally time consuming steps (1) \& (2) are independent from the measured data, but include the axisymmetric magnetic equilibrium field, which sets the helical symmetry, at the respective time during the discharge as well as the diagnostic and machine geometry. Only steps (3) \& (4) require the measured SXR image. Note further that the evaluation of the Tikhonov regularization, (step (3)), is instantaneous.
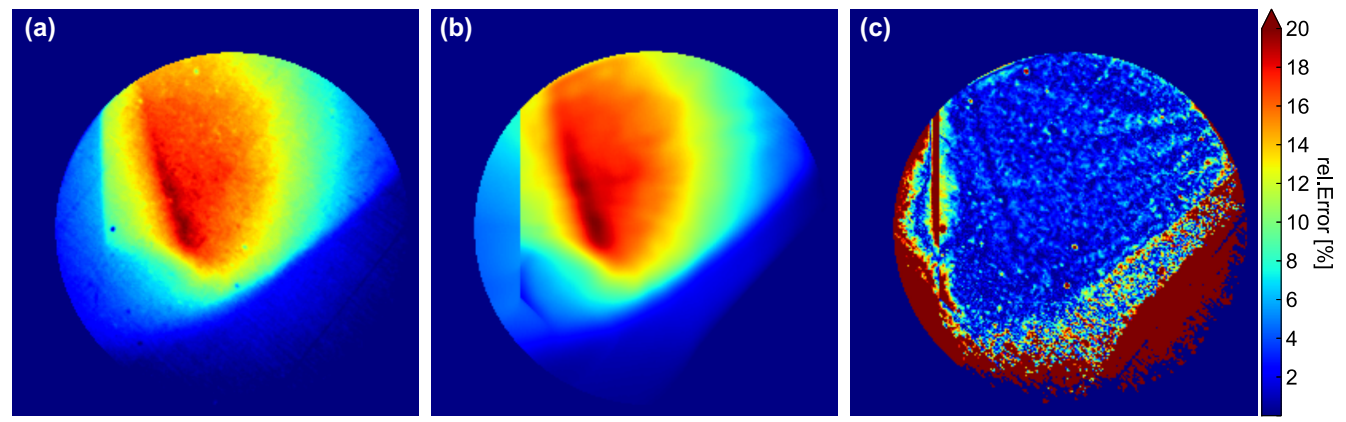

Figure 10: (color online) (a) Soft X-ray emission measured by the DIII-D SXRIS in discharge \#148712. The emission is spatially integrated over the view cone and temporarily integrated from $4020 \mathrm{~ms}$ to $4095 \mathrm{~ms}$.

(b) Recreated line-integrated image using Fig. 11(a).

(c) Relative error between (a) and (b).

To verify the success of the inversion, we can evaluate Eq. (1) using $\boldsymbol{\varepsilon}_{\lambda_{\text {opt }}}$ from step (4) and thereby reconstruct the SXRIS image. The result is shown in Fig. 10(b), which well agrees with the measured data. To quantify, we can calculate the relative error $\left\|\mathbf{s}_{S X R I S}-\mathbf{s}_{\lambda_{\text {opt }}}\right\| /\left\|\mathbf{s}_{S X R I S}\right\|$ between the measured and the reconstructed SXRIS images. Figure 10(c) confirms that inside the plasma volume the relative error is on the order of a few percent. The larger relative deviations occur only in regions of no signal or extremely low signal to noise, like the lower right corner or left of the centerpost boundary. Random pixel noise, as discussed in Sec. 4.2.2 is the source of error here. Image processing techniques, applied to Fig. 10(a) can potentially reduce the random noise. Note that a basic noise filter has already been applied to Fig. 10(a).

Figure 11(a) shows the optimum regularized solution $\varepsilon_{\lambda_{\text {opt }}}$. Several features are similar to the test image. The plasma bulk and boundary can clearly be identified. There is some low level emission in the scrape-off layer. Several structures can be seen inside the plasma in the steep gradient region of the pedestal. The nature of these structures is discussed in Ref. [15]. The 


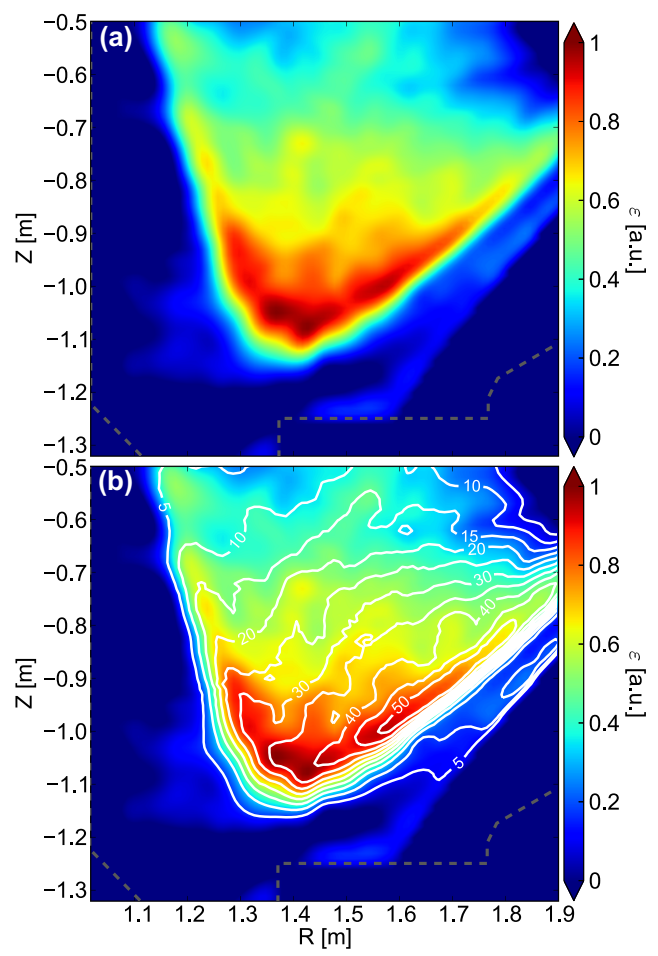

Figure 11: (color online) (a) Tikhonov regularization of Fig. 10(a) for optimum $\lambda=12.5$. Note that the image is cut of at $Z=-0.5$.

(b) Same as (a). The white contours show the levels of certainty in precent.

white contour lines in Fig. 11(b) indicate the level of certainty in percent, similar to Fig. 5(b). From the test image analysis we identified a minimum limit of $15 \%$ certainty to distinguish between real signal and inversion artifacts. The edge structures found in the inverted image are well inside this certainty limit. Using the Tikhonov regularization we are able to get good inversions which resolve the small scale structures in the plasma edge region we expect from plasma theories.

\section{Conclusions}

We developed an image inversion scheme for the SXRIS diagnostic at the DIII-D tokamak, to obtain the local SXR emission at a poloidal cross-section from the spatially line-integrated image, taken by the SXRIS camera. The scheme is based on the Tikhonov regularization method, since the inversion 
problem is generally ill-posed. We constructed a numerical algorithm, using the generalized singular value decomposition to determine a solution in dependence of a free regularization parameter. The latter has to be chosen carefully. Here we applied the L-curve method to determine the optimum regularization parameter, which is the best trade-off between the residual error and the applied side constraint.

To study the properties of the inversion scheme, we first considered a representative test image, which was constructed by assigning SXR emission to the simulated vacuum magnetic field structure, typical to DIII-D. A line-integrated image was obtained and subsequently inverted by our regularization algorithm. All features were reconstructed with excellent agreement to the test image. Only at the top boundary, inside the plasma core region, artifacts appeared. The inverted image has two main sources of error. Some emission zones get intersected by many sight-lines, while others only get intersected by few. Here, we define precision, see Eq. (22), as the relative amount of sight-line intersections for each emission zone, which is linked to the geometric setup and therefore a property of the inversion scheme. A precision analysis revealed that the top boundary suffers from very low sight-line coverage. We found that a precision above $15 \%$ is required to prevent artifacts. The second source of error is a low signal/noise ratio. The certainty of the inverted image is given by the product of precision and signal. We found that a certainty level of at least 15\% excludes artifacts, but includes the structures at the plasma edge.

Noise is found to be the most limiting issue, but sufficient regularization is still possible at noise levels of 10\%-15\% of the maximum signal.The optimum operational parameters for different noise levels are identified. We found that the zone resolution is optimal between $100 \mathrm{~m}^{-1}$ and $120 \mathrm{~m}^{-1}$. The pixel and the step resolution only have very weak influence on the inversion quality within the given limits that the total number of pixels must be larger than the total number of zones and that the step resolution must be larger than the zone resolution. We set the point of operation at $256 \times 256$ pixels, $100 \mathrm{~m}^{-1}$ zones and $200 \mathrm{~m}^{-1}$ steps. With this setting, an image inversion requires a minimum of 32 GB of memory and takes about 30-35 minutes on 16 cores with the bulk of time spent computing the GSVD.

Finally, we applied the inversion scheme to measured SXRIS data. The line-integrated SXRIS image is successfully inverted. We can identify key features in the inverted image. Since we now have a good quality reference, we want to optimize the presented procedures further in the future. Such 
optimizations could be numerical by increasing the computational efficiency through e.g. truncation of the GSVD, as well as physical by making use of $\varepsilon^{*}$, which has been set to zero throughout this paper. Preliminary tests show that $\varepsilon^{*}$ can be quite powerful and has to be chosen carefully. A detailed study is planned. By today, the presented method has become the standard technique to process SXRIS measurements at DIII-D for further physics analysis.

\section{Acknowledgements}

This work was supported by the US Department of Energy under DEAC05-00OR22725. Discussions with J.H. Harris and E. Chandler are gratefully acknowledged.

\section{References}

[1] G. Fuchs, Y. Miura, M. Mori, Soft x-ray tomography on tokamaks using flux coordinates, Plasma Phys. Control. Fusion 36 (1994) 307.

[2] M. Anton, H. Weisen, M. J. Dutch, W. von der Linden, F. Buhlmann, R. Chavan, B. Marletaz, P. Marmillod, P. Paris, X-ray tomography on the TCV tokamak, Plasma Phys. Control. Fusion 38 (1996) 1849.

[3] J. Wesson, Tokamaks, Clarendon Press, Oxford, 2004.

[4] S. Ohdachi, K. Toi, G. Fuchs, the LHD Experimental Group, Tangential SX imaging for visualization of fluctuations in toroidal plasmas, Plasma Fusion Res. 2 (2007) S1016.

[5] S. Ohdachi, K. Toi, G. Fuchs, TEXTOR Team, LHD Experimental Group, Magnetic islands observed by a fast-framing tangentially viewing soft x-ray camera on LHD and TEXTOR, Plasma Sci. Technol. 8 (2006) 45.

[6] L. C. Ingesson, B. Alper, H. Chen, A. W. Edwards, G. C. Fehmers, J. C. Fuchs, R. Giannella, R. D. Gill, L. Lauro-Taroni, M. Romanelli, Soft x-ray tomography during ELMs and impurity injection in JET, Nucl. Fusion 38 (1998) 1675.

[7] L. C. Ingesson, B. Alper, B. J. Peterson, J.-C. Vallet, Chapter 7 tomography diagnostics: Bolometry and soft-x-ray detection, Fusion Science and Technology 53 (2008) 528. 
[8] S. H. Lee, J. Kim, J. H. Lee, W. Choe, Modified Phillips-Tikhonov regularization for plasma tomography, Current Applied Physics 10 (2010) 893.

[9] J. Mlynar, V. Weinzettl, G. Bonheure, A. Murari, JET Contributors, Inversion techniques in the soft-x-ray tomography of fusion plasmas: Toward real-time applications, Fusion Science and Technology 58 (2010) 733.

[10] N. Iwama, H. Yoshida, H. Takimoto, Y. Shen, S. Takamura, T. Tsukishima, Phillips-Tikhonov regularization of plasma image reconstruction with the generalized cross validation, Applied Physics Letters 54 (1989) 502 .

[11] J. L. Luxon, A design retrospective of the DIII-D tokamak, Nucl. Fusion 42 (2002) 614.

[12] M. W. Shafer, D. J. Battaglia, E. A. Unterberg, T. E. Evans, D. L. Hillis, R. Maingi, 2D soft x-ray system on DIII-D for imaging the magnetic topology in the pedestal region, Rev. Sci. Instrum 81 (2010) 10 E534.

[13] J.-K. Park, A. H. Boozer, J. E. Menard, A. M. Garofalo, M. J. Schaffer, R. J. Hawryluk, S. M. Kaye, S. P. Gerhardt, S. A. Sabbagh, the NSTX Team, Importance of plasma response to nonaxisymmetric perturbations in tokamaks, Phys Plasmas 16 (2009) 056115.

[14] S. I. Itoh, K. Itoh, Model of H-mode in tokamaks, Nucl. Fusion 29 (1989) 1031.

[15] A. Wingen, N. M. Ferraro, M. W. Shafer, E. A. Unterberg, T. E. Evans, D. L. Hillis, P. B. Snyder, Impact of plasma response on plasma displacements in DIII-D during application of external 3-D perturbations, Nucl. Fusion 54 (2014) 064007.

[16] M. W. Shafer, D. J. Battaglia, E. A. Unterberg, J. M. Canik, T. E. Evans, J. H. Harris, S. J. Meitner, Soft x-ray imaging design and analysis methods on DIII-D, Plasma Fusion Res. 6 (2011) 2402041.

[17] A. N. Tikhonov, V. Y. Arsenin, Solutions of ill-posed problems, Wiley, New York, 1977. 
[18] G. H. Golub, P. C. Hansen, D. P. O'Leary, Tikhonov regularization and total least squares, SIAM J. MATRIX ANAL. APPL 21 (1997) 185-194.

[19] P. C. Hansen, Regularization tools - a matlab package for analysis and solution of discrete ill-posed problems, Numer. Algorithms 6 (1994) $1-35$.

[20] G. H. Golub, U. von Matt, Tikhonov regularization for large scale problems, in: G. H. Golub, S. H. Lui, F. Luk, R. Plemmons (Eds.), Scientific Computing, Springer, 1997, pp. 3-26.

[21] P.C. Hansen, D. O'Leary, The use of the L-curve in the regularization of discrete ill-posed problems, SIAM J. Sci. Comput. 14 (1993) 1487-1503.

[22] A. Wingen, O. Schmitz, T. E. Evans, K. H. Spatschek, Heat flux modelling using ion drift effects in DIII-D H-mode plasmas, Phys Plasmas 21 (2014) 012509. 